\title{
The optimal value of circular reserve for symmetrical hierarchical networks
}

\author{
M. B. Ahmadi ${ }^{1}$
}

(Received 9 October 2009; revised 22 September 2010)

\begin{abstract}
We consider the Symmetrical Hierarchical Network and analyse its survivability under uncertainty conditions. A formula calculates the optimum value of circular reserve of the symmetrical hierarchical network. We take into account the possibility of optimising the flow distribution after the fault. We suppose that the remaining capacity will be known. The guaranteed evaluation of the functional capability of the symmetrical hierarchical network assumes finding the worst distribution of the destruction.
\end{abstract}

\section{Contents}

1 Introduction

2 Competitive distribution of flows

http://anziamj . austms.org.au/ojs/index.php/ANZIAMJ/article/view/2497 gives this article, (c) Austral. Mathematical Soc. 2010. Published October 30, 2010. ISSN 1446-8735. (Print two pages per sheet of paper.) Copies of this article must not be made otherwise available on the internet; instead link directly to this URL for this article. 


\section{$1 \quad$ Introduction}

An arbitrary multi-commodity flow network (MFN) [15] is determined by two graphs, physical $G$ and logical $P$, on the same set $V$ of nodes. Edges $r_{k}$ of graph $\mathrm{G}$ mean physical lines of communications between the nodes from $\mathrm{V}$, and they are ascribed with non-negative numbers $y_{k}$ called the capacity of the edges $r_{k}, k=1, \ldots, n$. Edges $p_{i}, i \in M \stackrel{\text { def }}{=}\{1, \ldots, m\}$ of graph $P$ correspond to logical connections between certain pairs of nodes (source-sink pairs). This means that there are demands of flow transmission from one node to the other of $p_{i}$ through the edges of network graph $G$. Thus, each $p_{i}$ is specified by source-sink pair $\nu_{i}^{s}, v_{i}^{t} \in V$ and positive demand $d_{i}$.

A multi-commodity flow network is called symmetrical hierarchical network (SHN) if its logical graph has the structure of a star, that is, the source-sink pairs are given in the form $\left(v_{0}, v_{i}\right), i \in M$, with the common source $v_{0}$ and all demands are equal to $d$. The physical graph of a SHN usually repeats its logical structure and also is a star network: $G=\langle V, E\rangle$, where $V=$ $\left\{v_{0}, v_{1}, \ldots, v_{\mathrm{m}}\right\}$ and $\mathrm{E}=\left\{\boldsymbol{e}_{1}, e_{2}, \ldots, \boldsymbol{e}_{\mathrm{m}}\right\}$ where $\boldsymbol{e}_{\mathrm{i}}=\left(v_{0}, v_{\mathrm{i}}\right)$. We considered a formal hierarchical network [2].

Star topology is the most common type of network topology that is often used in computer networks and is typical for electricity networks. Star networks were proposed as an attractive alternative to the well-known hypercube models for interconnection networks. Extensive research has been performed that shows star networks are as versatile as hypercubes [5].

Sterbenz et al. [17] provided an architectural framework for resilience and 
survivability in communication networks and provides a survey of the disciplines that resilience encompasses, along with significant past failures of the network infrastructure.

Morehead and Noore [14] studied the resiliency of hierarchical networks and compared the results with random networks and scale-free networks. They used a load-based attack model and extended the dynamic model to cascading attacks.

Chang and Hwang [4] proposed an analytical model for evaluating the performance of adaptive hierarchical networks with multiple classes of traffic. They studied the reduced load approximation model for multi-rate loss networks, and then proposed a novel performance evaluation model for networks with hierarchical routing.

Manish Garg and Cole Smith [7] considered the design of a multi-commodity flow network, in which point-to-point demands are routed across the network subject to link capacity restrictions. Such a design must build enough capacity and diverse routing paths through the network to ensure that feasible multicommodity flows continue to exist, even when components of the network fail. They examine several methodologies to optimally design a minimum cost survivable network that continues to support a multi-commodity flow under any of a given set of failure scenarios, where each failure scenario consists of the simultaneous failure of multiple arcs.

Survivable system and survivable network have been designed and evaluated for many years. The concept of survivability is fundamental to many of the natural, social planning and engineering sciences, but its definition varies depending on the context of applications [8]. In many network design models, network survivability is defined as the ability of a network to maintain or restore an acceptable level of performance in the event of deterministic or random failures [18].

I summarise the many definitions of survivability as the following: Survivability is the system's ability to continuously deliver services in compliance with 
the given requirements in the presence of failures and other undesired events.

Network survivability is critical component of the global telecommunication infrastructure [16]. Network survivability was defined for an IP-over-WDM network as the ability of the network to recover from any single physical link failure [11]. Kan et al. [11] identified two criteria for satisfying in order to ensure network survivability:

1. the source and destination of every traffic demand must remain connected after any physical link failure; and

2. the spare capacity in the network must be sufficient to support all of the disrupted traffic.

The first criterion can be satisfied if the logical topology remains connected after any physical link failure. A routing was called survivable if any physical link failure leaves the logical topology connected. The problem of finding such a routing of the logical links on the physical topology has been studied [12]. However, the network must also have sufficient spare capacity to reroute the disrupted traffic. Determining where to place spare capacity in the network and how much spare capacity must be allocated to guarantee the restoration of the network against single link failures is called the spare capacity allocation problem.

Network survivability is an important requirement in high speed optical networks. The survivability of these networks from the failure of a single component such as a link or a node needs some typical approaches. Kalamani, Palaniswami and Nagarajan [10] considered double link failures in a hypercube optical network and presented three loopback methods suggested by the researchers for handling such failures. In the first two methods, two edgedisjoint backup paths are computed for each link for rerouting traffic when a pair of links fails. These methods require the identification of the failed links before recovery can be completed. The third method requires the precomputation of a single backup path and does not require link identification before recovery. Protection capacity, restorability, worst-case and average hop- 
length, worst-case and average number of connections are used as performance measures in their analysis.

Heegaard and Trivedi [9] considered the network's ability to survive major and minor failures in network infrastructure and service platforms that are caused by undesired events that might be external or internal. Survival means that the services provided comply with the requirement also in presence of failures.

Network survivability models considered by Heegaard and Trivedi [9] treat networks exposed to undesired events that cause links and nodes to fail, which are typically followed by a sudden change in the availability of network resources such as bandwidth of the transmission links, queuing positions (memory), and processor capacity. Gradually the resources are restored through rerouting and by restoration of the failed links and nodes, which results in restored performance.

Chen, Garg and Trivedi [6] propose a quantitative approach to evaluate network survivability. They perceive the network survivability as a composite measure consisting of both network failure duration and failure impact on the network.

The problem of the efficiency analysis of multiuser network systems was considered by Malashenko and Novikova [13] under conditions of nonrandom external perturbations, which decrease the edge capacity of the physical graph of the network. The formulation of the corresponding problem of the feasibility of multi-commodity flow networks for the known demand vector was proposed.

It is known that the physical structure of a star possesses poor properties of survivability. To create an additional circular structure connecting all the sinks may raise the survivability of SHN.

We considered the effectiveness of radial reserve to raise the survivability of SHN taking account of destruction of the main radial edges, and the radial reserve [1]. With the sufficient capacity of circular reserve for hierarchical 
network, we can obtain the maximum level of demand satisfaction [2]. We compared the efficiency of sufficient circular and radial reserve to raise the survivability of SHN [3]. Here we find the optimal value of circular reserve of SHN that guarantee the maximum level of demand satisfaction.

Denote by $\overline{\mathrm{G}}$ the SHN with additional circular reserve, that is, $\overline{\mathrm{G}}=\langle\mathrm{V}, \overline{\mathrm{E}}\rangle$, $\overline{\mathrm{E}}=\mathrm{E} \cup \mathrm{E}^{0}, \mathrm{E}^{0}=\left\{e_{1}^{0}, e_{2}^{0}, \ldots, e_{\mathrm{m}}^{0}\right\}$, where $e_{i}^{0}=\left(v_{i}, v_{i+1}\right), i \neq m$, and $e_{m}^{0}=$ $\left(v_{\mathrm{m}}, v_{1}\right)$. Let $\mathrm{c}=\left(\mathrm{c}_{1}, \ldots, \mathrm{c}_{\mathrm{m}}\right)$ be a capacity vector for circular edges of $\overline{\mathrm{G}}$.

Let $z_{j}$ be the amount flow between nodes $v_{0}$ and $v_{j}$ and $f_{i}^{j}$ denote the share of flow $z_{j}$ between $v_{0}$ and $v_{j}$ which passes by edge $\left(v_{0}, v_{i}\right)$. The variables $f_{i}^{j \rightarrow}$ and $f_{i}^{j \leftarrow}$ denotes the share of the flow $z_{j}$ between $v_{0}$ and $v_{j}$ that passes by circular edges clockwise and counter-clockwise respectively.

In these variables the flow constraint conditions for SHN are

$$
\begin{aligned}
& \sum_{i=1}^{m} f_{i}^{j}=z_{j} \quad \text { for all } j \in M, \\
& f_{i}^{j}=f_{i}^{j \rightarrow}+f_{i}^{j \leftarrow} \quad \text { for all } i, j \in M, i \neq j, \quad f_{j}^{j \rightarrow}=f_{j}^{j \leftarrow}=0,
\end{aligned}
$$

and the capacity constraints for sHN for all $i \in M$ are

$$
\begin{aligned}
& \sum_{j=1}^{m} f_{i}^{j} \leqslant y_{i}, \\
& \sum_{j=1}^{i-1}\left(\sum_{l=j+1}^{i-1} f_{l}^{j \rightarrow}+\sum_{l=1}^{j-1} f_{l}^{j \leftarrow}+\sum_{l=i}^{m} f_{l}^{j \leftarrow}\right) \\
& +\sum_{j=i}^{m}\left(\sum_{l=1}^{i-1} f_{l}^{j \rightarrow}+\sum_{l=j+1}^{m} f_{l}^{j \rightarrow}+\sum_{l=i}^{j-1} f_{l}^{j \leftarrow}\right) \leqslant c_{i} .
\end{aligned}
$$

Denote by $f \in R_{+}^{3 m^{2}}$ the set of flow variables $f_{i}^{j}, f_{i}^{j \rightarrow}$ and $f_{i}^{j \leftarrow}, i \in M$ and $j \in M$, that satisfy (1). The constraints (1), (2) and (3) define the polyhedron of feasible flow distributions

$$
F(y, c)=\{f \geqslant 0 \mid \text { subject to(1), (2), (3) }\} .
$$




\section{Competitive distribution of flows}

Let $\overline{\mathrm{d}}=(\mathrm{d}, \ldots, \mathrm{d})$ and $\mathrm{c}=\left(\mathrm{c}_{1}, \ldots \mathrm{c}_{\mathrm{m}}\right)$ be the initial capacity vectors for radial and circular edges of SHN respectively, and $\bar{y}=(\overline{\mathrm{d}}, \mathrm{c})$.

Denote by $Z(\bar{y})$ the set of all feasible multiflows $z=\left(z_{1}, \ldots, z_{m}\right)$ in the network with capacity vector $\bar{y}$, that is,

$$
\mathrm{Z}(\overline{\mathrm{y}})=\{z \geqslant 0 \mid \text { there exists } \mathrm{f} \in \mathrm{F}(\overline{\mathrm{y}}): z=z(\mathrm{f})\},
$$

and define

$$
\theta_{0}=\theta_{0}(\bar{y})=\max _{f \in F(\bar{y})} \min _{i \in M} \frac{z_{i}(f)}{d} .
$$

The value of $\theta_{0}$ shows the measure of effectiveness functioning of SHN.

If $\mathrm{f}^{0}$ be the optimal solution of $(5)$, then $\mathrm{f}^{0}$ is called the competitive distribution of flows.

In the case $\mathrm{m}=1,(5)$ is a well known problem of flow maximisation. For $m>1$ it formalises the concurrent flow problem. A multi-flow achieves demand satisfaction if it ships an amount of each commodity equal to its demand from its source to its sink while obeying the capacity constraint. This corresponds to $\theta_{0} \geqslant 1$. Otherwise, an arbitrary concurrent flow distribution $f^{0}$ (a maximiser in (5)) may be not the best for certain network users.

The function $\theta_{\gamma}^{g}(\bar{y})$ denotes the guaranteed level of demand satisfaction depending on reserve capacity $\mathrm{c}$ and demands $\mathrm{d}$ and is defined as

$$
\theta_{\gamma}^{g}(\bar{y})=\min _{y \in Y(\gamma)} \theta_{0}(y, c)=\min _{y \in Y(\gamma)} \max _{f \in F(\bar{y})} \min _{i \in M} \frac{z_{i}(f)}{d},
$$

where

$$
Y(\gamma)=\left\{y \geqslant 0 \mid \sum_{i=1}^{m} y_{i}=(1-\gamma) m d, \quad y_{i} \leqslant d \quad \text { for all } i \in M\right\} .
$$


Here $\gamma \in(0,1)$ is a parameter which characterises the power of network destruction. It indicates what part of the total capacity of radial edges may be lost.

The Survivability of SHN is defined by $\theta_{\gamma}^{g}(\bar{y})$. Note that $\theta_{\gamma}^{g}(\bar{y}) \leqslant 1-\gamma$, $\gamma \in(0,1)$.

In this article a formula is given for calculating the circular reserve value which is necessary and sufficient to guarantee the level $\theta_{\gamma}^{\mathrm{g}}(\overline{\mathrm{y}})=1-\gamma, \gamma \in(0,1)$.

We introduce $\mathrm{C}(\gamma)$, the set of possible alternatives of a sufficient ring redundancy,

$$
\mathrm{C}(\gamma)=\left\{\mathrm{c}=\left(\mathrm{c}_{1}, \ldots, \mathrm{c}_{\mathrm{m}}\right) \mid(1-\gamma) \overline{\mathrm{d}} \in \mathrm{Z}(\mathrm{y}, \mathrm{c}) \text { for all } \mathrm{y} \in \mathrm{Y}(\gamma)\right\} .
$$

Theorem 1 Let $\overline{\mathrm{d}}=(\mathrm{d}, \ldots, \mathrm{d})$ and $\mathrm{c}=\left(\mathrm{c}_{1}, \ldots, \mathrm{c}_{\mathrm{m}}\right)$ be the initial capacity vectors of radial and circular edges of SHN respectively, and $\mathrm{t}^{0}$ be the optimum value of circular reserve for SHN that guarantees the level $\theta_{\gamma}^{\mathrm{g}}(\overline{\mathrm{y}})=1-\gamma$, $\gamma \in(0,1)$. Then

$$
t^{0}=\min _{c \in C(\gamma)} \max _{i \in M} c_{i}= \begin{cases}\frac{1}{2}(m-\lceil(1-\gamma) m\rceil) d(1-\gamma), & l>1-\gamma, \\ \frac{1}{2}\lfloor(1-\gamma) m\rfloor d \gamma, & l \leqslant 1-\gamma,\end{cases}
$$

where $\mathrm{l}=(1-\gamma) \mathrm{m}-\lfloor(1-\gamma) \mathrm{m}\rfloor$ and $\lfloor\mathrm{a}\rfloor(\lceil\mathrm{a}\rceil)$ is the nearest integer value less (greater) than or equal to a.

Proof: We first show that

there exists $c^{0}=\left(c_{1}^{0}, \ldots c_{m}^{0}\right) \in C(\gamma)$ : for all $i \in M \quad c_{i}^{0} \leqslant t^{0}$.

This means that $t^{0}$ is a sufficient capacity for circular edges in order to guarantee the maximum level of demand satisfaction. Let $c^{0}=\left(t^{0}, \ldots, t^{0}\right)$. We must prove that

for all $y \in Y(\gamma)$ there exists $f \in F\left(y, c^{0}\right): z(f)=\bar{d}(1-\gamma)$. 
Assume that $y=\left(y_{1}, \ldots, y_{m}\right) \in Y(\gamma)$. Define $f \in R_{+}^{3 m^{2}}$, the set of variables $f_{i}^{j}, f_{i}^{j \rightarrow}$ and $f_{i}^{j \leftarrow}, i, j \in M$,

$$
\begin{aligned}
& f_{i}^{j \rightarrow}=f_{i}^{j \leftarrow}= \begin{cases}-t_{i} t_{j}\left[2 \sum_{r \in I^{+}} t_{r}\right]^{-1}, & i \in I^{+}, j \in I^{-}, \\
0, & \text { otherwise, }\end{cases} \\
& f_{i}^{j}=f_{i}^{j \rightarrow}+f_{i}^{j \leftarrow}, \quad i \neq j, \\
& f_{j}^{j}= \begin{cases}y_{j}, & j \in I^{-}, \\
d(1-\gamma), & j \in I^{+},\end{cases}
\end{aligned}
$$

where

$$
\mathrm{t}_{\mathrm{i}}=\mathrm{y}_{\mathrm{i}}-\mathrm{d}(1-\gamma), \quad \mathrm{I}^{+}=\left\{\mathrm{i} \mid \mathrm{t}_{\mathrm{i}}>0\right\}, \quad \mathrm{I}^{-}=\mathrm{M} \backslash \mathrm{I}^{+} .
$$

Now we show that $z(f)=\bar{d}(1-\gamma)$.

$$
\begin{aligned}
& z_{j}=\sum_{l=1}^{m} f_{l}^{j}=\sum_{l=1, l \neq j}^{m}\left(f_{l}^{j \rightarrow}+f_{l}^{j \leftarrow}\right)+f_{j}^{j} \\
& =\sum_{l \in I^{+}, l \neq j}\left(f_{l}^{j \rightarrow}+f_{l}^{j \leftarrow}\right)+\sum_{l \in I^{-}, l \neq j}\left(f_{l}^{j \rightarrow}+f_{l}^{j \leftarrow}\right)+f_{j}^{j} \\
& = \begin{cases}d(1-\gamma), & j \in I^{+}, \\
\sum_{l \in I^{+}}-t_{l} t_{j}\left[\sum_{r \in I^{+}} t_{r}\right]^{-1}+y_{j}, & j \in I^{-},\end{cases} \\
& = \begin{cases}d(1-\gamma), & j \in \mathrm{I}^{+}, \\
-\mathrm{t}_{\mathrm{j}}+\mathrm{y}_{\mathrm{j}}, & j \in \mathrm{I}^{-},\end{cases} \\
& = \begin{cases}d(1-\gamma), & j \in I^{+}, \\
d(1-\gamma) m, & j \in I^{-},\end{cases} \\
& =d(1-\gamma) \text { for all } j \in M \text {. }
\end{aligned}
$$

Now we show that $\sum_{i \in I^{+}} t_{i}=-\sum_{i \in I^{-}} t_{i}$.

$$
y \in Y(\gamma) \Rightarrow \sum_{i \in M} y_{i}=(1-\gamma) m d \Rightarrow \sum_{i \in M}\left(y_{i}-(1-\gamma) d\right)=0
$$




$$
\Rightarrow \sum_{i \in M} t_{i}=0 \Rightarrow \sum_{i \in I^{+}} t_{i}=-\sum_{i \in I^{-}} t_{i} .
$$

Let us now check the capacity constraints on the radial and circular edges of SHN.

$$
\begin{aligned}
& \sum_{j \in M, j \neq l}\left(f_{l}^{j \rightarrow}+f_{l}^{j \leftarrow}\right)+f_{l}^{l} \stackrel{(9)-(11)}{=} \begin{cases}\sum_{j \in I^{-}}-t_{l} t_{j}\left[\sum_{r \in I^{+}} t_{r}\right]^{-1}+f_{l}^{l}, & l \in I^{+}, \\
f_{l}^{l}, & l \in I^{-},\end{cases} \\
& \stackrel{(13)}{=} \begin{cases}t_{l}+f_{l}^{l}, & l \in I^{+}, \\
f_{l}^{l}, & l \in I^{-},\end{cases} \\
& = \begin{cases}y_{l}-d(1-\gamma)+d(1-\gamma), & l \in I^{+}, \\
y_{l}, & i \in I^{-},\end{cases} \\
& =y_{l} \quad \text { for all } l \in M \text {. }
\end{aligned}
$$

We verified the capacity constraints on the radial edges.

By (3) and (9), the capacity constraints on the ring becomes

$$
\sum_{j=1}^{i-1}\left(\sum_{l=1, l \neq j}^{m} f_{l}^{j \rightarrow}\right)+\sum_{j=i}^{m}\left(\sum_{l=1, l \neq j}^{m} f_{l}^{j \rightarrow}\right) \leqslant c_{i} \quad \text { for all } i \in M,
$$

and hence

$$
\sum_{j=1}^{m} \sum_{l=1, l \neq j}^{m} f_{l}^{j \rightarrow} \leqslant c_{i} \quad \text { for all } i \in M .
$$

Thus for the checking of the capacity constraints on the ring, we must show that

$$
\sum_{j=1}^{m} \sum_{l=1, l \neq j}^{m} f_{l}^{j \rightarrow} \leqslant c_{i}=t^{0} \quad \text { for all } i \in M, \quad \text { for all } y \in Y(\gamma) .
$$

By (9) and (13)

$$
\sum_{j=1}^{m} \sum_{l=1, l \neq j}^{m} f_{l}^{j \rightarrow}=\sum_{j \in I^{-}} \sum_{l \in I^{+}}-\frac{t_{l} t_{j}}{2 \sum_{r \in I^{+}} t_{r}}=\frac{1}{2} \sum_{l \in I^{+}} t_{l} .
$$


To prove (14), we show that

$$
\begin{aligned}
& \max _{y \in Y(\gamma)} \sum_{l \in \mathrm{I}^{+}} \mathrm{t}_{l}=2 \mathrm{t}^{0} . \\
& \max _{y \in Y(\gamma)} \sum_{l \in \mathrm{I}^{+}} \mathrm{t}_{l}=\max _{y \in Y(\gamma)} \sum_{i \in M}\left[y_{i}-\mathrm{d}(1-\gamma)\right]^{+},
\end{aligned}
$$

where $[\cdot]^{+}=\max (0, \cdot)$.

Define $\psi_{l}(y)=\left[y_{l}-d(1-\gamma)\right]^{+}$. For all $l \in M, \psi_{l}(y)$ is a convex function and therefore $\sum_{l \in M} \psi_{l}$ is also a convex function.

Let

$$
\begin{aligned}
& \mathrm{I}_{1}=\left\{\mathrm{i} \mid \mathrm{y}_{\mathrm{i}}=\mathrm{d}, \mathrm{y} \in \mathrm{Y}(\gamma)\right\}, \\
& \mathrm{I}_{2}=\left\{\mathrm{i} \mid \mathrm{y}_{\mathrm{i}}=0, \mathrm{y} \in \mathrm{Y}(\gamma)\right\}, \\
& \mathrm{I}_{3}=\left\{\mathrm{i} \mid 0<\mathrm{y}_{\mathrm{i}}<\mathrm{d}, \mathrm{y} \in \mathrm{Y}(\gamma)\right\}
\end{aligned}
$$

Note that, $y \in Y(\gamma)$ is an extreme point of $Y(\gamma)$ if and only if $y \in S \stackrel{\text { def }}{=}\{y \in$ $\left.Y(\gamma)|| I_{3} \mid \leqslant 1\right\}$.

Another presentation of $S$ is $S=\left\{y \in Y(\gamma) \mid y=\left(d, \ldots, d, y_{q}, 0, \ldots, 0\right)\right.$ or vectors given from any permutation of $y\}$, where $0 \leqslant y_{q} \leqslant d$,

$$
\begin{aligned}
& y_{q}=m d(1-\gamma)-\lfloor m(1-\gamma)\rfloor d \\
& q=\left|I_{1}\right|+1=\lfloor(1-\gamma) m\rfloor+1 .
\end{aligned}
$$

Now we show that the value of $\sum_{i=1}^{m} \psi_{i}(y)$ for all $y$ in $S$ is the same. Assume that this is not so. Then two cases are possible:
1. $y_{q}>d(1-\gamma)$;
2. $y_{q} \leqslant d(1-\gamma)$. 
In Case 1 for all $y \in S$ we have

$$
\begin{aligned}
\sum_{i \in M} \psi_{i}(y) & =\sum_{i \in M}\left[y_{i}-d(1-\gamma)\right]^{+} \\
& =\left|I_{1}\right|(d-d(1-\gamma))+y_{q}-d(1-\gamma) \\
& =\lfloor(1-\gamma) m\rfloor d \gamma+m d(1-\gamma)-\lfloor(1-\gamma) m\rfloor d-d(1-\gamma) \\
& =(m-1) d(1-\gamma)-\lfloor(1-\gamma) m\rfloor d(1-\gamma) \\
& =(m-1-\lfloor(1-\gamma) m\rfloor) d(1-\gamma) \\
& =(m-\lceil(1-\gamma) m\rceil) d(1-\gamma) .
\end{aligned}
$$

In Case 2 for all $y \in S$

$$
\begin{aligned}
\sum_{i \in M} \psi_{i}(y) & =\sum_{i \in M}\left[y_{i}-d(1-\gamma)\right]^{+}=\left|I_{1}\right| d \gamma \\
& =\lfloor(1-\gamma) m\rfloor d \gamma .
\end{aligned}
$$

Clearly, the value of $\sum_{i \in M} \psi_{i}(y)$ for any $y \in S$ in Cases 1 and 2 is not dependent on $y$ and this value is the same for all $y$ belong to $S$. Since $\sum_{i \in M} \psi_{i}(y)$ is a convex function and $Y(\gamma)$ is a convex polyhedron, then the optimal solution for (17) occur at any extreme point of $Y(\gamma)$.

By (17), (18), (20) and (21) we obtain

$$
\begin{aligned}
\max _{y \in Y(\gamma)} \sum_{l \in I^{+}} t_{l} & =\left\{\begin{array}{l}
(m-\lceil(1-\gamma) \mathrm{m}\rceil) \mathrm{d}(1-\gamma) \\
\text { if }(1-\gamma) \mathrm{m}-\lfloor(1-\gamma) \mathrm{m}\rfloor>1-\gamma, \\
\lfloor(1-\gamma) \mathrm{m}\rfloor \mathrm{d} \gamma \\
\text { if }(1-\gamma) \mathrm{m}-\lfloor(1-\gamma) \mathrm{m}\rfloor \leqslant 1-\gamma,
\end{array}\right. \\
= & 2 \mathrm{t}^{0}
\end{aligned}
$$

By (14) and (22) we verified the capacity constraints on the ring (circular edges) and therefore

$$
c=\left(t^{0}, \ldots, t^{0}\right) \in C(\gamma)
$$


Now we show for all $c=\left(c_{1}, \ldots, c_{m}\right) \in C(\gamma)$ there exists $l \in M: c_{l} \geqslant t^{0}$. Let

$$
c=\left(c_{1}, \ldots, c_{m}\right) \in C(\gamma), \quad \tilde{y}=\left(d, \ldots, d, \tilde{y}_{q}, 0, \ldots, 0\right) \in Y(\gamma) \text {, }
$$

where $\mathbf{q}=[(1-\gamma) \mathrm{m}]+1, \tilde{y}_{\mathrm{q}}=(1-\gamma) \mathrm{md}-\lfloor(1-\gamma) \mathrm{m}\rfloor \mathrm{d}$. Two cases are possible

1. $\tilde{y}_{q}>d(1-\gamma)$;

2. $\tilde{y}_{q} \leqslant d(1-\gamma)$.

In Case 1 since $c \in C(\gamma)$, therefore for all $f \in F(\tilde{y}, c)$ from (3), for $i=q+1$,

$$
\begin{aligned}
& \sum_{j=1}^{q}\left(\sum_{l=j+1}^{q} f_{l}^{j \rightarrow}+\sum_{l=1}^{j-1} f_{l}^{j \leftarrow}+\sum_{l=q+1}^{m} f_{l}^{j \leftarrow}\right) \\
& +\sum_{j=q+1}^{m}\left(\sum_{l=1}^{q} f_{l}^{j \rightarrow}+\sum_{l=j+1}^{m} f_{l}^{j \rightarrow}+\sum_{l=q+1}^{j-1} f_{l}^{j \leftarrow}\right) \leqslant c_{q+1} .
\end{aligned}
$$

Since $f_{l}^{j \rightarrow}, f_{l}^{j \leftarrow}$ for all $l, j$ are positive,

$$
\sum_{j=q+1}^{m} \sum_{l=1}^{q} f_{l}^{j \rightarrow} \leqslant c_{q+1} .
$$

Similarly, the below inequality can be obtained for $i=1$.

$$
\sum_{j=q+1}^{m} \sum_{l=1}^{q} f_{l}^{j \leftarrow} \leqslant c_{1} .
$$

Thus

$$
\sum_{j=q+1}^{m} \sum_{l=1}^{q}\left(f_{l}^{j \rightarrow}+f_{l}^{j \leftarrow}\right) \leqslant c_{1}+c_{q+1} .
$$


Since $\tilde{y}=\left(d, \ldots, d, \tilde{y}_{q}, 0, \ldots, 0\right), \tilde{y}_{q}>d(1-\gamma)$, the amount of flow that can pass by edge $l, l=1, \ldots, q$, (that is, $\sum_{j=q+1}^{m} f_{l}^{j}$ ) is equal to $y_{l}-d(1-\gamma)$ and

$$
\begin{aligned}
& \sum_{j=q+1}^{m} \sum_{l=1}^{q}\left(f_{l}^{j \rightarrow}+f_{l}^{j \leftarrow}\right) \\
= & \sum_{j=q+1}^{m} \sum_{l=1}^{q} f_{l}^{j}=\sum_{l=1}^{q}\left(y_{l}-d(1-\gamma)\right) \\
= & \sum_{l=1}^{q-1}(d-d(1-\gamma))+\tilde{y}_{q}-d(1-\gamma) \\
= & (q-1) d \gamma+\tilde{y}_{q}-d(1-\gamma) \\
= & \lfloor(1-\gamma) m\rfloor d \gamma+(1-\gamma) m d-\lfloor(1-\gamma) m\rfloor d-d(1-\gamma) \\
= & d(1-\gamma)((m-1)-\lfloor(1-\gamma) m\rfloor) \\
= & d(1-\gamma)(m-\lceil(1-\gamma) m\rceil) .
\end{aligned}
$$

From (24) and (25) we obtain

$$
\mathrm{d}(1-\gamma)(\mathrm{m}-\lceil(1-\gamma) \mathrm{m}\rceil) \leqslant \mathrm{c}_{1}+\mathrm{c}_{\mathrm{q}+1} .
$$

Thus either

$$
c_{1} \geqslant \frac{d(1-\gamma)(m-\lceil(1-\gamma) m\rceil)}{2}
$$

or

$$
c_{q+1} \geqslant \frac{d(1-\gamma)(m-\lceil(1-\gamma) m\rceil)}{2},
$$

hence there exists $l \in M: c_{l} \geqslant t^{0}$.

In Case 2, similar to Case 1, we derive the two inequalities

$$
\sum_{j=q}^{m} \sum_{l=1}^{q-1} f_{l}^{j \rightarrow} \leqslant c_{q}, \quad \text { and } \quad \sum_{j=q}^{m} \sum_{l=1}^{q-1} f_{l}^{j \leftarrow} \leqslant c_{1} .
$$


Thus

$$
\sum_{j=q}^{m} \sum_{l=1}^{q-1}\left(f_{l}^{j \rightarrow}+f_{l}^{j \leftarrow}\right) \leqslant c_{1}+c_{q} .
$$

On the other hand,

$$
\begin{aligned}
\sum_{j=q}^{m} \sum_{l=1}^{q-1}\left(f_{l}^{j \rightarrow}+f_{l}^{j \leftarrow}\right) & =\sum_{l=1}^{q-1}(d-d(1-\gamma))=(q-1) \gamma d \\
& =\lfloor(1-\gamma) m\rfloor \gamma d .
\end{aligned}
$$

From (26) and (27) either

$$
c_{1} \geqslant \frac{\lfloor(1-\gamma) \mathrm{m}\rfloor \mathrm{d} \gamma}{2} \text { or } \quad c_{\mathrm{q}} \geqslant \frac{\lfloor(1-\gamma) \mathrm{m}\rfloor \mathrm{d} \gamma}{2} .
$$

Hence in Case 2, as well as, Case 1

$$
\text { there exists } l \in M: c_{l} \geqslant t^{0},
$$

and therefore for all $c \in C(\gamma)$ there exists $l \in M: c_{l} \geqslant t^{0}$. This completes the proof of the theorem.

\section{$3 \quad$ Numerical example}

Let $\overline{\mathrm{d}}=(10,10,10,10)$ be the initial capacity vector of SHN, and $\gamma=0.1$. In this case we have $\mathrm{m}=4$ and

$$
\begin{aligned}
Y(0.1)= & \left\{y=\left(y_{1}, y_{2}, y_{3}, y_{4}\right) \mid \sum_{i=1}^{4} y_{i}=(1-0.1)(4)(10)=36\right. \\
& \left.0 \leqslant y_{i} \leqslant 10, i=1,2,3,4\right\}, \\
l= & (1-\gamma) m-\lfloor(1-\gamma) m\rfloor=0.9(4)-\lfloor 0.9(4)\rfloor=3.6-\lfloor 3.6\rfloor=0.6 .
\end{aligned}
$$


Since $l<1-\gamma=0.9$, the optimal value for circular reserve is

$$
t^{0}=\frac{\lfloor 0.9(4)\rfloor 10(0.1)}{2}=1.5 .
$$

If we take $c=(1.5,1.5,1.5,1.5)$ as a capacity vector of circular edges, then we transmit flow to each sink through the remaining radial edges and circular reserve that guaranteed $\theta_{\gamma}^{\mathrm{g}}(\overline{\mathrm{d}}, \mathrm{c})=0.9$. For example, suppose that $\mathrm{y}=$ $(6,10,10,10)$. In this case

$$
\sum_{i=1}^{4} y_{i}=36=(1-0.1) 4(10) \Rightarrow y \in Y(0.1) \text {. }
$$

We take

$$
\begin{aligned}
& f_{1}^{1}=6, \quad f_{2}^{2}=f_{3}^{3}=f_{4}^{4}=9, \\
& f_{2}^{1 \rightarrow}=0, \quad f_{2}^{1 \leftarrow}=1, \quad f_{3}^{1 \rightarrow}=0.5, \quad f_{3}^{1 \leftarrow}=0.5, \quad f_{4}^{1 \rightarrow}=1, \quad f_{4}^{1 \leftarrow}=0, \\
& f_{i}^{j \rightarrow}=f_{i}^{j \leftarrow}=0 \quad \text { for } i=1,2,3,4 \text { and } j=2,3,4 .
\end{aligned}
$$

The maximum flow transmitted by each circular edge is less or equal to 1.5. In addition, we have

$$
\begin{aligned}
z_{1}=\sum_{i=1}^{4} f_{i}^{1} & =f_{1}^{1}+\left(f_{2}^{1 \rightarrow}+f_{2}^{1 \leftarrow}\right)+\left(f_{3}^{1 \rightarrow}+f_{3}^{1 \leftarrow}\right)+\left(f_{4}^{1 \rightarrow}+f_{4}^{1 \leftarrow}\right) \\
& =6+(0+1)+(0.5+0.5)+(1+0)=9,
\end{aligned}
$$

and similarly, we obtain

$$
z_{2}=z_{3}=z_{4}=9 .
$$

Thus $\theta_{0}(y, c)=\min _{i} z_{i} / 10=0.9$.

\section{Conclusion}

Physical star structures have poor survivability characteristics. By creating a supplementary ring structure with sufficient capacity, that links all the 
subordinate nodes, we can duplicate the messages in the event of loss of communication between the centre and one or more nodes and guarantee the maximum level of effectiveness of the functioning of the SHN.

\section{References}

[1] Ahmadi M. B., The Survivability of Symmetrical Hierarchical Networks with Radial Reserve, Journal Application and Applied Mathematics: An International Journal (AAM), 3(2008) 30-41. E26

[2] Ahmadi M. B., Malashenko Yu. E., Novikova N. M., Research on survivability of a hierarchical network, J. Vestnik Moscow state university serial 15, computational mathematics and cybernetics 3(2001) 18-23 (in Russian). E23, E27

[3] Ahmadi M. B., A comparison of effectiveness of circular and radial reserve in survivability of symmetrical hierarchical networks, J. WSEAS transaction on computer, Issue 6, 3(2004) 1764-1766. E27

[4] Chang B.-J., Hwang R.-H., Modeling and analyzing the performance of adaptive hierarchical networks, Information Sciences 176 (2006) 522-549. E24

[5] Chen Ch, Chen J., Nearly Optimal One-to-Many Parallel Routing in Star Networks, IEEE Transactions on Parallel and Distributed Systems, Issue 12, 8(1997) 1196-1202. E23

[6] Chen D., Garg S., Trivedi K. S., Network Survivability Performance Evaluation: A Quantitative Approach with Applications in Wireless Adhoc Networks, Proceedings of the 5th ACM international workshop on Modeling analysis and simulation of wireless and mobile systems, (2002) 61-68. E26 
[7] Garg M., Smith J. C., Models and algorithms for the design of survivable multicommodity flownetworks with general failure scenarios, Omega 36 (2008) 1057-1071. E24

[8] Grubesic T. H., Murray A. T. Spatial-historical landscapes of telecommunication network survivability, Telecommunication policy, (2005) 801-820. E24

[9] Heegaard P. E., Trivedi K. S., Network survivability modeling, Computer Networks 53 (2009) 1215-1234. E26

[10] Kalamani K, Palaniswami S, and Nagarajan N, Network Survivability for a Regular WDM Optical Network from Double- Link Failures Using Greedy Algorithm, International Journal of Recent Trends in Engineering, 1 (2009) 478-482. E25

[11] Kan D. D., Narula-Tam A., Modiano E., Lightpath Routing and Capacity Assignment for Survivable IP-over-WDM Networks, 7 th International Workshop on the Design of Reliable Communication Networks Washington, DC, USA (2009) 37-44. E25

[12] Lee K., Modiano E., Cross-layer Survivability in WDM based Networks, Proc. IEEE INFOCOM'09, Rio de Janeiro, Brazil, April (2009). E25

[13] Malashenko Yu. E., Novikova N. M., The Analysis of Multi user Network Systems under Uncertainty, J. of Computer and Systems Sciences International, 38(1999) 589-601. E26

[14] Morehead R. , Noore A., Novel hybrid mitigation strategy for improving the resiliency of hierarchical networks subjected to attacks, Physica $A$ 378 (2007) 603-612. E24

[15] Phillips D., Garcia-Dias A., Fundamentals of network analysis, (Englewood Cliffs, N. J. : Prentice-Hall,1981). E23 
[16] Shi J., Foneska J. P., Traffic Based Survivability Analysis of Telecommunications Networks,Proc. IEEE Globe-com 95, 2(1995) 936-940. E25

[17] Sterbenz J. P. G. , Hutchison D., Cetinskaya E. K., Jabbar A., Rohrer J. P., Schller M., Smith P., Resilience and survivability in communication networks: Strategies, principles, and survey of disciplines, Computer Networks 54 (2010) 1245-1265. E23

[18] Wu T., Kolar D. J., Cardwell R. H., Survivable networks architectures for broad-band fiber optic networks: Model and performance comparison, Journal of Lightwave Technology, (1998)1698-1709. E24

\section{Author address}

1. M. B. Ahmadi, Dept. of Math., College of Sciences, Shiraz University, 71454 Shiraz, IRAN.

mailto:mbahmadi@shirazu.ac.ir 